\title{
Analysis of Unsteady MHD Thin Film Flow of a Third Grade Fluid with Heat Transfer down an Inclined Plane
}

\section{Aiyesimi YM1 ${ }^{1}$, Okedayo $\mathrm{GT}^{2}$ and Lawal OW'*}

${ }^{1}$ Department of Mathematics, Federal University of Technology Minna, Niger State, Nigeria

${ }^{2}$ Department of Mathematics, Ondo State University of Science and Technology Okitipupa, Ondo State, Nigeria

\begin{abstract}
An investigation is made for unsteady MHD thin film flow of a third grade fluid down an inclined plane with no slip boundary condition. The governing nonlinear partial differential equations involved are reduced to linear partial differential equations using regular perturbation method. The resulting equations were solved using method of separation of variable and eigen functions expansion. The solutions obtained were examined and discussed graphically. It is interesting to find that the variation of the velocity and temperature profile with the magnetic field parameter and gravitational parameter depends on time.
\end{abstract}

Keywords: Eigen value, MHD thin film, Homotopy

\section{Introduction}

In recent years considerable interest has been developed in the study of the flow of non- Newtonian fluid down an inclined plane because of its important applications in science, engineering and technology. Examples of their application can be found in ink-jet print, polymer processing, silvering processing, plasma enhanced chemical vapour deposition as well as in magnetorheological thin film deposition. Because of the complexity of non-Newtonian fluids, it is very difficult to analyse a single model that exhibits all its properties. The normal stress differences is describe in second grade of non-Newtonian fluid, but it cannot predict shear thinning or thickening properties due to its constant apparent viscosity. The third grade fluid model attempt to include such characteristics of viscoelastics fluids. Many authors have successfully analysed the nonlinear differential equation governing the flow of a third grade fluid [1-3]. He [4-6] introduced a new perturbation method which is a combination of regular perturbation method and homotopy as used in topology. Siddiqui et al. [7], Khan and Mahmood [8], Aiyesimi et al. [9] and other authors recently applied this newly introduced homotopy perturbation method to solved nonlinear differential equation that governing the steady thin film flow of a third grade fluid down an inclined plane.

In this paper, we obtain analytical solution of the unsteady MHD thin film flow problem for a third grade fluid down an inclined plane with no slip boundary condition as formulated in Aiyesimi et al. [10]. The governing nonlinear differential equations involved are reduced to linear differential equations using regular perturbation method. The resulting equations were solved using method of separation of variable and eigen function expansion.

\section{Problem Formulation}

In Aiyesimi et al. [10], we studied MHD thin film flow of a third grade fluid down an inclined plane. We assumed that the flow was unsteady, the ambient air was stationary, surface tension negligible and the thin film is of uniform thickness $\delta$ [11-15]. The whole system was subjected to magnetic field and in the absence of pressure gradient, we obtained

$$
\begin{aligned}
\rho \frac{\partial u}{\partial t} & =\mu \frac{\partial^{2} u}{\partial y^{2}}+\alpha_{1} \frac{\partial^{3} u}{\partial y^{2} \partial t}+6\left(\beta_{2}+\beta_{3}\right)\left(\frac{\partial u}{\partial y}\right)^{2} \frac{\partial^{2} u}{\partial y^{2}} \\
& -\sigma B_{0}^{2} u+\rho g \sin \theta
\end{aligned}
$$

$$
0<\delta<1 \quad t>0
$$

with the boundary condition

$$
\begin{array}{ll}
u(y, t)=0 \text { at } y=0 \quad t>0 \\
\frac{\partial u(y, t)}{\partial y}=0 \text { at } y=\delta \quad t>0
\end{array}
$$

and initial condition

$$
u(y, 0)=0 \quad 0<y<\delta
$$

where $\rho$ is the density of the fluid, $u$ is the fluid velocity, $B_{0}$ represent induced magnetic field, $\sigma$ is the electrical conductivity, $\alpha_{1}, \beta_{2}$ and $\beta_{3}$ are the material constant and $\theta$ indicate angle of inclination.

The energy equation for the thermodynamically compatible third grade fluid with viscous dissipation, work done due to deformation and Joule heating was given as

$$
\begin{aligned}
& \rho c_{p} \frac{\partial T}{\partial t}=K \frac{\partial^{2} T}{\partial y^{2}}+\mu\left(\frac{\partial u}{\partial y}\right)^{2}+\alpha_{1} \frac{\partial u}{\partial y} \frac{\partial^{2} u}{\partial y \partial t} \\
&+2\left(\beta_{2}+\beta_{3}\right)\left(\frac{\partial u}{\partial y}\right)^{4}+\sigma B_{0}^{2} u^{2} \\
& 0<\delta<1 \quad t>0,
\end{aligned}
$$

with boundary condition

$T(y, t)=T_{w}$ at $y=0 \quad t>0$

*Corresponding author: Lawal OW, Department of Mathematics, Federal University of Technology Minna, Niger State, Nigeria, Tel: 07032270305, 234(0)7098809476; E-mail: waheedlawal207@yahoo.com

Received January 17, 2014; Accepted February 12, 2014; Published February 17,2014

Citation: Aiyesimi YM, Okedayo GT, Lawal OW (2014) Analysis of Unsteady MHD Thin Film Flow of a Third Grade Fluid with Heat Transfer down an Inclined Plane. J Appl Computat Math 3: 152. doi:10.4172/2168-9679.1000152

Copyright: @ 2014 Aiyesimi YM, et al. This is an open-access article distributed under the terms of the Creative Commons Attribution License, which permits unrestricted use, distribution, and reproduction in any medium, provided the original author and source are credited. 
and initial condition

$$
T(y, 0)=0 \quad 0<y<\delta
$$

where $c_{p}$ and $k$ are respectively, specific heat capacity and thermal conductivity of the fluid. $T$ is the temperature of the fluid, $T_{w}$ is the temperature of the plane and $T_{\delta}$ is the temperature of the ambient fluid.

We define the following non-dimensional quantities

$u=\mu \bar{u}, \quad y=\bar{y} \delta, \quad \eta=\frac{\mu y}{\delta}, \bar{t}=\frac{\mu^{2} t}{\delta}$,

$T=\bar{T}\left(T_{\delta}-T_{w}\right)$

$\alpha=\frac{\alpha_{1} \mu^{2}}{\rho \delta^{2}}$, is the second grade parameter

$\beta=\frac{\left(\beta_{2}+\beta_{3}\right) \mu^{4}}{\rho \delta^{3}}$, is the third grade parameter

$\mathrm{M}=\frac{\delta \sigma B_{0}^{2}}{\rho \mu^{2}}$, is the magnetic parameter

$K=\frac{\delta g \sin \theta}{\mu^{3}}$, is the gravitational parameter

$P_{r}=\frac{\mu \rho c_{p}}{K}$, is the Prandth number

$\mathrm{E}_{c}=\frac{\mu^{4}}{c_{p}\left(T_{\infty}-T_{w}\right)}$, is the Eckert number

$\lambda=\frac{\alpha}{\beta}$, is the fluid grade ratio

In terms of these above non-dimensional variables and parameters, equation (1)-(8) are written as (as we dropped "hats" for convenience)

$$
\begin{aligned}
\frac{\partial u}{\partial t}= & \frac{\partial^{2} u}{\partial \eta^{2}}+\beta\left[\lambda \frac{\partial^{3} u}{\partial \eta^{2} \partial t}+6\left(\frac{\partial u}{\partial \eta}\right)^{2} \frac{\partial^{2} u}{\partial \eta^{2}}\right], 0<\eta<1, t>0 \\
& -M u+K=0
\end{aligned}
$$

with the boundary and initial condition

$$
\begin{array}{ll}
u(0, t)=0 & t>0 \\
\frac{\partial u(1, t)}{\partial \eta}=0 & t>0 \\
u(\eta, 0)=0 & 0<\eta<1
\end{array}
$$

For energy equation, we have

$$
\begin{aligned}
\frac{\partial T}{\partial t} & =\frac{1}{P_{r}} \frac{\partial^{2} T}{\partial \eta^{2}}+E_{c}\left(\frac{\partial u}{\partial \eta}\right)^{2}+\beta\left[\begin{array}{c}
\lambda \mathrm{E}_{c} \frac{\partial u}{\partial \eta} \frac{\partial^{2} u}{\partial \eta \partial t} \\
+2 \mathrm{E}_{c}\left(\frac{\partial u}{\partial \eta}\right)^{4}
\end{array}\right] \quad 0<\eta<1, t>0 \\
& +M \mathrm{E}_{c} u^{2}
\end{aligned}
$$

with the boundary and initial condition

$$
T(0, t)=0 \quad t>0
$$

$$
\begin{array}{lc}
T(1, t)=1 & t>0 \\
T(\eta, 0)=0 & 0<\eta<1
\end{array}
$$

\section{Solutions of the Problem}

The basic requirement for the application of solution by perturbation method is the existence of small or large parameter in equation (10), therefore let assumed $\varepsilon=\beta$ as a small parameter and expand $u(\eta, t)$ in the Poincare-type series of the form

$$
u(\eta, t)=u_{0}(\eta, t)+\varepsilon u_{1}(\eta, t)
$$

Substituting equation (18) into equation (10) - (13) and collect the like terms base on the power of , we have

Zeroth-order problem

$$
\begin{aligned}
& \frac{\partial u_{0}}{\partial t}=\frac{\partial^{2} u_{0}}{\partial \eta^{2}}-M u_{0}+K, 0<\eta<1, t>0 \\
& u_{0}(0, t)=0, \quad \frac{\partial u_{0}(1, t)}{\partial \eta}=0 \quad t>0 \\
& u_{0}(\eta, 0)=0, \quad 0<\eta<1
\end{aligned}
$$

First order problem

$$
\begin{aligned}
& \frac{\partial u_{1}}{\partial t}=\frac{\partial u_{1}}{\partial \eta^{2}}+\lambda \frac{\partial^{3} u_{0}}{\partial \eta^{2} \partial t}+6\left(\frac{\partial u_{0}}{\partial \eta}\right)^{2} \frac{\partial^{2} u_{0}}{\partial \eta^{2}}-M u_{1}, 0<\eta<1, t>0 \\
& u_{1}(0, t)=0, \quad \frac{\partial u_{1}(1, t)}{\partial \eta}=0 \quad t>0 \\
& u_{1}(\eta, 0)=0, \quad 0<\eta<1
\end{aligned}
$$

In order to solve equation (19) which is Initial Boundary Value Problem (IBVP) together with equation (20) and (21), let assume a solution of the form

$$
u_{0}(\eta, t)=u_{0 \infty}(\eta)+v_{0}(\eta, t)
$$

where $u_{0 \infty}$ is the equilibrium solution of equation (19)-(21) which satisfies the Boundary Value Problem (BVP)

$$
\begin{aligned}
& \frac{\partial^{2} u_{0 \infty}}{\partial \eta^{2}}-M u_{0 \infty}+K=0 \\
& u_{0 \infty}(0)=0 \quad \frac{\partial u_{0 \infty}(1)}{\partial \eta}=0
\end{aligned}
$$

The solution of equation (26) with boundary condition (27) is given as

$$
u_{0 \infty}(\eta)=\frac{-K \cosh (\eta \sqrt{M}-\sqrt{M})+K \cosh (\sqrt{M})}{M \cosh (\sqrt{M})}
$$

Substituting equation (28) into (25) to give

$$
u_{0}(\eta, t)=v_{0}(\eta, t)-\frac{-K \cosh (\eta \sqrt{M}-\sqrt{M})+K \cosh (\sqrt{M})}{M \cosh \sqrt{M}}
$$

also

Substituting equation (29) into equation (19) to gives

$$
\frac{\partial v_{0}}{\partial t}=\frac{\partial^{2} v_{0}}{\partial \eta^{2}}-M v_{0}, 0<\eta<1, t>0 \text { (29) }
$$


The boundary and initial condition (20) and (21) becomes

$$
\begin{aligned}
& v_{0}(0, t)=0, \quad \frac{\partial v_{0}(1, t)}{\partial \eta}=0, \quad t>0 \\
& v_{0}(\eta, 0)=-\frac{-K \cosh (\eta \sqrt{M}-\sqrt{M})+K \cosh (\sqrt{M})}{M \cosh (\sqrt{M})}
\end{aligned}
$$

Equation (30) and boundary condition (31) are linear and homogeneous, which have a solution of the form

$$
v_{0}(\eta, t)=\sum_{n=1}^{\infty} b_{n} \sin \left[\frac{(2 n-1)}{2} \pi \eta\right] \ell^{-\frac{(2 n-1)^{2} \pi^{2} t}{4}-M t}
$$

Substituting equation (28) and (33) into equation (25) to gives

$$
\begin{aligned}
u_{0}(\eta, t)= & \frac{-K \cosh (\eta \sqrt{M}-\sqrt{M})+K \cosh (\sqrt{M})}{M \cosh \sqrt{M}} \\
& +\sum_{n=1}^{\infty} b_{n} \sin \left[\frac{(2 n-1)}{2} \pi \eta\right] \ell^{-\frac{(2 n-1)^{2}}{4} \pi^{2} t-M t}
\end{aligned}
$$

where

$$
b_{n}=2 \int_{0}^{1}-\frac{-K \cos (\eta \sqrt{M}-\sqrt{M})+K \cosh (\sqrt{M})}{M \cosh (\sqrt{M})} \sin \frac{(2 n-1)}{2} \pi \eta d \eta \quad n=1,2, \ldots \ldots
$$

Solving first order problem, equation (22) can written as

$$
\frac{\partial u_{1}}{\partial t}=\frac{\partial^{2} u_{1}}{\partial \eta^{2}}-M u_{1}+q_{0}(\eta, t), 0<\eta<1, t>0
$$

where

$$
q_{0}(\eta, t)=\lambda \frac{\partial^{3} u_{0}}{\partial \eta^{2} \partial t}+6\left(\frac{\partial u_{0}}{\partial \eta}\right)^{2} \frac{\partial^{2} u_{0}}{\partial \eta^{2}}
$$

The eigenvalues and eigenfunctions of the corresponding homogeneous problem of equation (35) when $q_{0}(\eta, t)=0$ are respectively

$$
\begin{aligned}
& \lambda_{1 n}=\frac{(2 n-1)^{2} \pi^{2}}{4}+M \\
& Y_{1 n}(\eta)=\sin \left[\frac{(2 n-1) \pi \eta}{2}\right], \quad n=1,2, \ldots . .
\end{aligned}
$$

Since $\left\{Y_{1 n}\right\}_{n=1}^{\infty}$ is a complete sets, we may consider for the solution of equation (35) an expansion of the form

$$
u_{1}(\eta, t)=\sum_{n=1}^{\infty} c_{1 n}(t) Y_{1 n}(\eta)
$$

substituting equation (33) and (38) into (18) to obtain

$$
\begin{aligned}
u(\eta, t) & =\frac{-k \cosh (\eta \sqrt{M}-\sqrt{M})+k \cosh (\sqrt{M})}{M \cosh (\sqrt{M})} \\
& +\sum_{n=1}^{\infty} b_{n} \sin \left[\frac{(2 n-1) \pi}{2} \eta\right] \ell^{-\frac{(2 n-1)^{2} \pi^{2} t}{4}} \\
& +\varepsilon \sum_{n=1}^{\infty} c_{1 n}(t) Y_{1 n}(\eta)
\end{aligned}
$$

Therefore when $n=1$

$$
\begin{aligned}
u(\eta, t) & =\frac{-k \cosh (\eta \sqrt{M}-\sqrt{M})+k \cosh (\sqrt{M})}{M \cosh (\sqrt{M})} \\
& +b_{1} \sin \left(\frac{\pi \eta}{2}\right) \ell^{-\frac{\pi^{2} t}{4}-M t} \\
& +\sum_{n=2}^{\infty} b_{n} \sin \left[\frac{(2 n-1) \pi}{2} \eta\right] \ell^{-\frac{(2 n-1)^{2} \pi^{2} t}{4}} \\
& +\varepsilon\left[c_{11}(t) Y_{11}(\eta)+\sum_{n=2}^{\infty} c_{1 n}(t) Y_{1 n}(\eta)\right]
\end{aligned}
$$

where

$$
\begin{aligned}
& c_{11}(t)=\left(\begin{array}{l}
\frac{a_{4} \ell^{-t\left(\frac{1}{4} \pi^{2}+M\right)}}{-\frac{\pi^{2}}{4}-M}+\frac{a_{1} \ell^{t\left(\frac{1}{\pi^{2}}+M\right)}}{\frac{\pi^{2}}{4}+M} \frac{a_{2} \ell^{-t\left(\frac{1}{2} \pi^{2}+2 M\right)}}{-\frac{\pi^{2}}{2}-2 M}+a_{3} t-\frac{a_{4}}{-\frac{a^{2}}{4}-M}-\frac{a_{1}}{\frac{\pi^{2}}{4}+M} \\
-\frac{\pi^{2}}{2}-2 M
\end{array}\right) \ell^{-t\left(\frac{1}{4} \pi^{2}+M\right)} \\
& Y_{11}(\eta)=\sin \left[\frac{\pi \eta}{2}\right] \\
& b_{1}=\frac{4 K\left(-4 \ell^{2 \sqrt{M}} M-4 M\right)}{\pi M\left(\pi^{2}+4 M+\ell^{2 \sqrt{M}} \pi^{2}+4 \ell^{2 \sqrt{M}} M\right)}
\end{aligned}
$$

For $n=2$

$$
\begin{aligned}
u(\eta, t) & =\frac{-k \cosh (\eta \sqrt{M}-\sqrt{M})+k \cosh \sqrt{M}}{M \cosh \sqrt{M}} \\
& +b_{1} \sin \left(\frac{\pi \eta}{2}\right) \ell^{-\frac{\pi^{2} t}{4}-M t} \\
& +b_{2} \sin \left(\frac{3 \pi \eta}{2}\right) \ell^{-\frac{9 \pi^{2} t}{4}-M t} \\
& +\sum_{n=3}^{\infty} b_{n} \sin \left[\frac{(2 n-1) \pi}{2} \eta\right] \ell^{-\frac{(2 n-1)^{2} \pi^{2} t}{4}} \\
& +\varepsilon\left[\begin{array}{l}
\left.c_{11}(t) Y_{11}(\eta)+c_{12}(t) Y_{12}(\eta)\right] \\
+\sum_{n=3}^{\infty} c_{1 n}(t) Y_{1 n}(\eta)
\end{array}\right.
\end{aligned}
$$

$$
\begin{aligned}
& c_{12}(t)=\left[a_{8} t+\frac{a_{5} \ell^{t / 4\left(\pi^{2}+4 M\right)}}{\frac{9}{4} \pi^{2}+M}+\frac{a_{6} \ell^{-\frac{1}{2} t\left(9 \pi^{2}+4 M\right)}}{-\frac{9}{2} \pi^{2}-2 M}\right. \\
& +\frac{a_{7} \ell^{-\frac{t}{4}\left(9 \pi^{2}+4 M\right)}}{-\frac{9}{4} \pi^{2}-M}-\frac{a_{5}}{\frac{9}{4} \pi^{2}+M} \\
& \left.-\frac{a_{6}}{-\frac{9}{2} \pi^{2}-2 M}-\frac{a_{7}}{-\frac{9}{4} \pi^{2}-M}\right] e^{-\frac{t}{4}\left(9 \pi^{2}+4 M\right)}
\end{aligned}
$$

$Y_{12}=\sin \left(\frac{3}{2} \pi \eta\right)$ 


$$
b_{2}=\frac{4 k\left(-4 \ell^{2 \sqrt{M}} M-4 M\right)}{\pi M\left(12 M+27 \pi^{2}+27 \ell^{2 \sqrt{M}} \pi^{2}+12 \ell^{2 \sqrt{M}} M\right)}
$$

where $a_{i}(i=1,2, \ldots 8)$ are constant.

Next, we find the approximate solution for energy equation (14) with boundary and initial condition (15)-(17) by chosen $u(\eta, t)$ from equation (40) when $n=1$ and $\varepsilon$ which is equal to $\beta$ is turn to zero (i.e $\varepsilon=\beta \rightarrow 0$ ) as temperature increases.

Therefore equation (14) becomes

$$
\frac{\partial T}{\partial t}=\frac{1}{\operatorname{Pr}} \frac{\partial^{2} T}{\partial \eta^{2}}+q_{1 T}(\eta, t)
$$

where

$$
q_{1 T}(\eta, t)=E_{c}\left(\frac{\partial u}{\partial \eta}\right)^{2}+M E_{c} u^{2}
$$

also, we choose

$$
\begin{aligned}
u(\eta, t) & =\frac{-K \cosh (\eta \sqrt{M}-\sqrt{M})+K \cosh (\sqrt{M})}{M \cosh (\sqrt{M})} \\
+ & \frac{4 K\left(-4 \ell^{2 \sqrt{M}} M-4 M\right) \ell^{-\frac{\pi^{2} t}{4}-M t} \sin \left(\frac{\pi}{2} \eta\right)}{\pi M\left(\pi^{2}+4 M+\ell^{2 \sqrt{M}} \pi^{2}+4 \ell^{2 \sqrt{M}} M\right)}
\end{aligned}
$$

Let

$$
T(\eta, t)=\varphi(\eta)+\theta(\eta, t)
$$

Substituting (45) into (42), (15) - (17) to obtain the following

$$
\begin{aligned}
& \frac{\partial^{2} \phi(\eta)}{\partial \eta^{2}}=0 \\
& \phi(0)=0, \quad \phi(1)=1
\end{aligned}
$$

also

$$
\begin{aligned}
& \frac{\partial \theta}{\partial t}=\frac{1}{\operatorname{Pr}} \frac{\partial^{2} \theta}{\partial \eta^{2}}+q_{1 T}(\eta, t), 0<\eta<1, t>0 \\
& \theta(0, t)=0, \quad \theta(1, t)=0, \quad t>0 \\
& \theta(\eta, 0)=-\phi(\eta), \quad 0<\eta<1
\end{aligned}
$$

Solving equation (46) with (47), we have

$$
\phi(\eta)=\eta
$$

To solve equation (48) together with (49)-(51), the eigenvalues and eigenfunctions of the corresponding homogeneous problem of equation (48) when $q_{1 T}(\eta, t)=0$ are respectively

$$
\lambda_{r T}=(r \pi)^{2} \quad Y_{r T}=\sin (r \pi \eta)
$$

where $r=1,2, \ldots$

Since $\left\{Y_{r T}\right\}_{r=1}^{\infty}$ is a complete sets, we may consider for the solution an expansion of the form

$$
\theta(\eta, t)=\sum_{r=1}^{\infty} c_{r T}(t) Y_{r T}(\eta)
$$

when $r=1$

$$
\begin{aligned}
c_{1 T}(t)= & \frac{a_{9} \operatorname{Pr} \ell^{\frac{\pi^{2} t}{\operatorname{Pr}}}}{\pi^{2}}-\frac{4 a_{10} \operatorname{Pr} \ell^{-\frac{\frac{1}{4}^{t}\left(-4 \pi^{2}+\pi^{2} \operatorname{Pr}+4 M \operatorname{Pr}\right)}{\operatorname{Pr}}}}{-4 \pi^{2}+\pi^{2} \operatorname{Pr}+4 M \operatorname{Pr}} \\
- & \frac{2 a_{11} \operatorname{Pr} \ell^{-\frac{1}{2} t\left(-2 \pi^{2}+\pi^{2} \operatorname{Pr}+4 M \operatorname{Pr}\right)}}{-2 \pi^{2}+\pi^{2} \operatorname{Pr}+4 M \operatorname{Pr}}-\frac{2}{\pi}-\frac{a_{10} \operatorname{Pr}}{\pi^{2}} \\
& +\frac{4 a_{10} \operatorname{Pr}}{-4 \pi^{2}+\pi^{2} \operatorname{Pr}+4 M \operatorname{Pr}} \\
& \left.+\frac{2 a_{11} \operatorname{Pr}}{-2 \pi^{2}+\pi^{2} \operatorname{Pr}+4 M \operatorname{Pr}}\right] \ell^{\frac{-\pi^{2} t}{\operatorname{Pr}}} \\
Y_{1 T}(\eta)= & \sin (\pi \eta)
\end{aligned}
$$

Therefore

$$
\begin{aligned}
\theta(\eta, t) & =c_{1 T}(t) Y_{1 T}(\eta)+\sum_{r=2}^{\infty} c_{r T}(t) Y_{r T}(\eta) \\
= & {\left[\frac{a_{9} \operatorname{Pr} \ell^{\frac{\pi^{2} t}{\operatorname{Pr}}}}{\pi^{2}}-\frac{4 a_{10} \operatorname{Pr} \ell^{-\frac{\frac{1}{4}^{t}\left(-4 \pi^{2}+\pi^{2} \mathrm{Pr}+4 M \mathrm{Pr}_{\mathrm{r}}\right)}{\operatorname{Pr}}}}{-4 \pi^{2}+\pi^{2} \operatorname{Pr}+4 M \operatorname{Pr}}\right.} \\
& -\frac{2 a_{11} \operatorname{Pr} \ell^{-\frac{1}{2} t\left(-2 \pi^{2}+\pi^{2} \mathrm{Pr}+4 M \mathrm{Pr}\right)}}{-2 \pi^{2}+\pi^{2} \operatorname{Pr}+4 M \operatorname{Pr}}-\frac{2}{\pi}-\frac{a_{10} \operatorname{Pr}}{\pi^{2}}(52) \\
+ & \frac{4 a_{10} \operatorname{Pr}}{-4 \pi^{2}+\pi^{2} \operatorname{Pr}+4 M \operatorname{Pr}} \\
+ & \left.\frac{2 a_{11} \operatorname{Pr}}{-2 \pi^{2}+\pi^{2} \operatorname{Pr}+4 M \operatorname{Pr}}\right] \ell^{\frac{-\pi^{2} t}{\mathrm{Pr}}} \sin (\pi \eta) \\
+ & \sum_{r=2}^{\infty} c_{r T}(t) Y_{r T}(\eta)
\end{aligned}
$$

Also, when $r=2$, then

$$
\begin{aligned}
& c \quad(t)=\left[-\frac{a_{12} \operatorname{Pr} \ell^{\overline{\mathrm{Pr}}}}{\pi^{2}}-\frac{2 a_{13} \operatorname{Pr} \ell^{\frac{-t\left(-8 \pi^{2}+\pi^{2} \mathrm{Pr}+4 M \mathrm{Pr}\right)}{\operatorname{Pr}}}}{-8 \pi^{2}+\pi^{2} \operatorname{Pr}+4 \operatorname{Pr}}\right. \\
& -\frac{4{ }_{14} \operatorname{Pr} \frac{--t\left(-16 \pi^{2}+\pi^{2} \operatorname{Pr}+4 M \mathrm{Pr}\right)}{\operatorname{Pr}}}{-16 \pi^{2}+\pi^{2} \operatorname{Pr}+4 \operatorname{Pr}}+\frac{1}{\pi}-\frac{1}{{ }^{2}} \operatorname{Pr} \\
& \frac{2{ }_{13} \operatorname{Pr}}{-8 \pi^{2}+\pi^{2} \operatorname{Pr}+4} \mathrm{Pr} \\
& \left.\frac{4{ }_{14} \operatorname{Pr}}{-16 \pi^{2}+\pi^{2} \operatorname{Pr}+4} \operatorname{Pr}\right] \frac{}{{ }^{\mathrm{Pr}}}
\end{aligned}
$$

$Y_{2 T}(\eta)=\sin (2 \pi \eta)$ 
Then

$$
\begin{gathered}
\theta(\eta, t)=c_{1 T}(t) Y_{1 T}(\eta)+c_{2 T}(t) Y_{2 T}(\eta) \\
+\sum_{n=3}^{\infty} c_{r T}(t) Y_{r T}(\eta)
\end{gathered}
$$

Therefore

$$
\begin{aligned}
T(\eta, t) & =\eta+c_{1 T}(t) Y_{1 T}(\eta)+c_{2 T}(t) Y_{2 T}(\eta) \\
& +\sum_{n=3}^{\infty} c_{r T}(t) Y_{r T}(\eta)
\end{aligned}
$$

Again, when $n=2$ and $\varepsilon=\beta \rightarrow$, we choose

$$
\begin{aligned}
& u(\eta, t)=\frac{-K \cosh (\eta \sqrt{M}-\sqrt{M})+K \cosh (\sqrt{M})}{M \cosh (\sqrt{M})} \\
& +\frac{4 K\left(-4 \ell^{2 \sqrt{M}} M-4 M\right) \ell^{-\frac{\pi^{2} t}{4}-M t} \sin \left(\frac{\pi}{2} \eta\right)}{\pi M\left(\pi^{2}+4 M+\ell^{2 \sqrt{M}} \pi^{2}+4 \ell^{2 \sqrt{M}} M\right)} \\
& \frac{+4\left(-4 \ell^{2 \sqrt{M}} M-4 M\right) K \sin \left(\frac{3}{2} \pi \eta\right) \ell^{-\frac{9}{4} \pi^{2} t-M t}}{\pi M\left(12 M+27 \pi^{2}+27 \pi \ell^{2 \sqrt{M}} \pi^{2}+12 \ell^{2 \sqrt{M}} M\right)}
\end{aligned}
$$

then we obtained

$$
T(\eta, t)=\bar{\varphi}(\eta)+\bar{\theta}(\eta, t)
$$

where

$$
\bar{\phi}(\eta)=\eta
$$

and

$$
\bar{\theta}(\eta, t)=\sum_{r=1}^{\infty} c_{r \bar{T}}(t) Y_{r \bar{T}}(\eta)
$$

when $r=1$

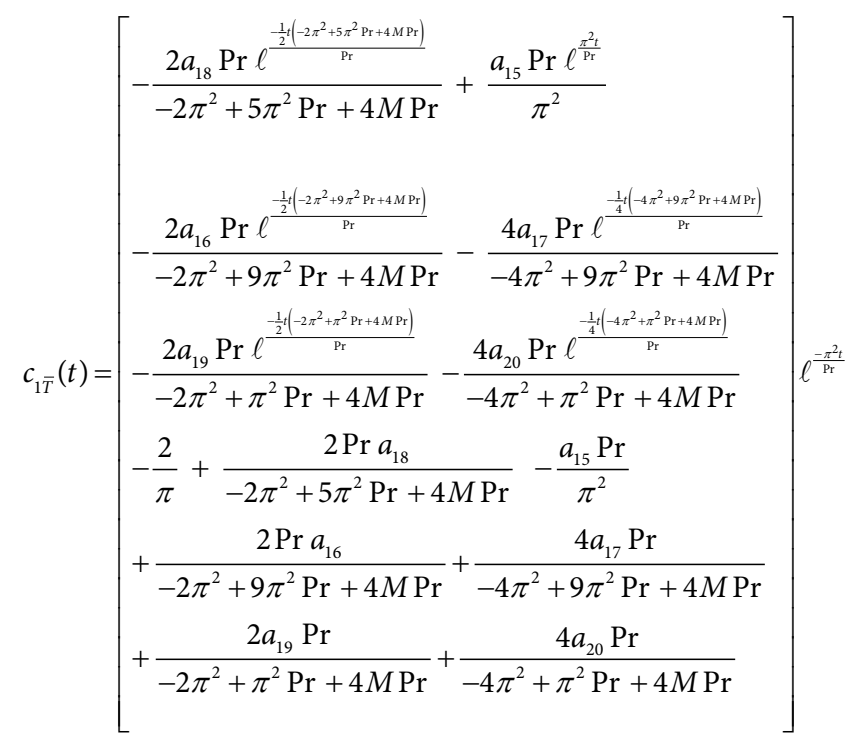

$Y_{1 \bar{T}}=\sin (\pi \eta)$, when $r=2$

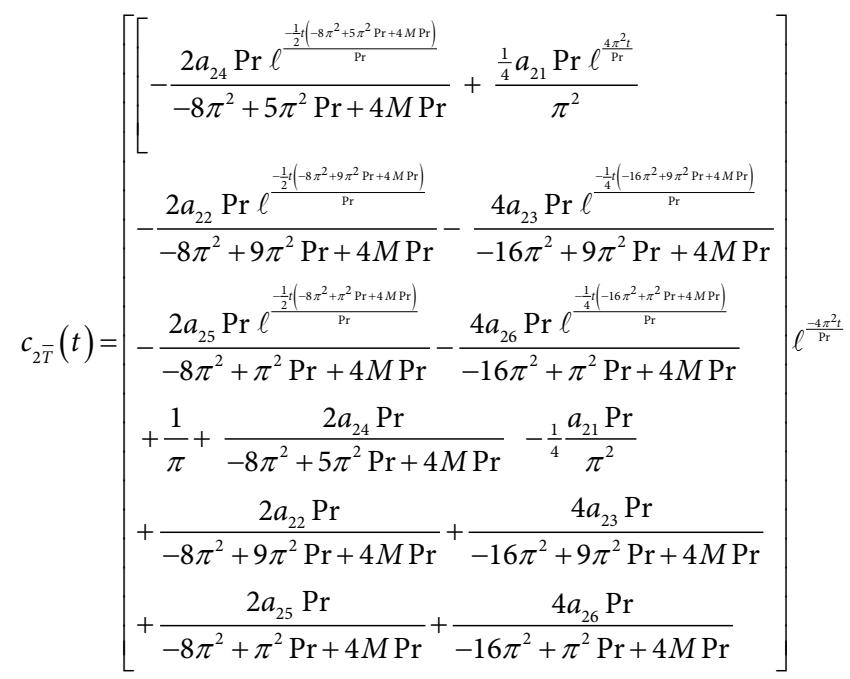$$
Y_{2 \bar{T}}=\sin (2 \pi \eta)
$$

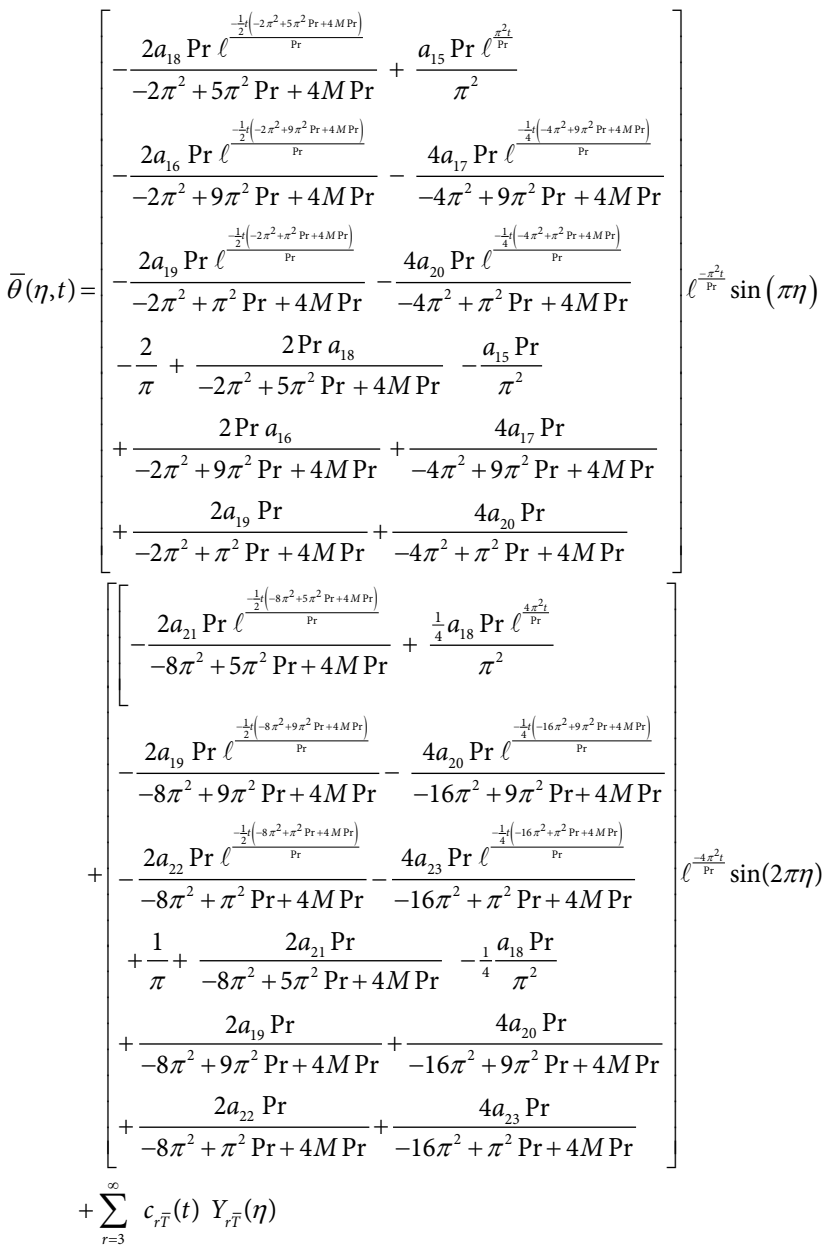

and $a_{i}(i=9,10,11, \ldots \ldots, 24)$ are constant and can be calculated through simple computation.

\section{Results and Discussion}

In this section, we discuss the behaviour of the velocity and temperature profile $u$ and $T$ of unsteady MHD thin film flow of a third 
grade fluid as it move down an inclined plane in the absence of slip parameter.

Figure 1a-1d and Figure 2a-2d shows the time development of velocity profile $u$ and temperature profile $T$ for various value of time $t$ and for $M=1,5,10$ and 25.

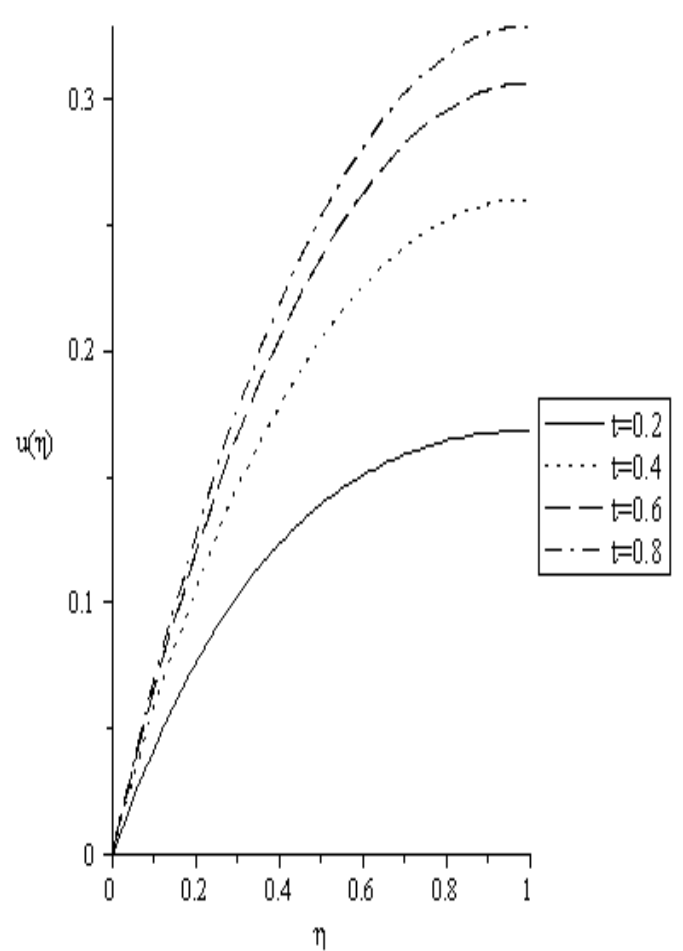

(a) $M=1$

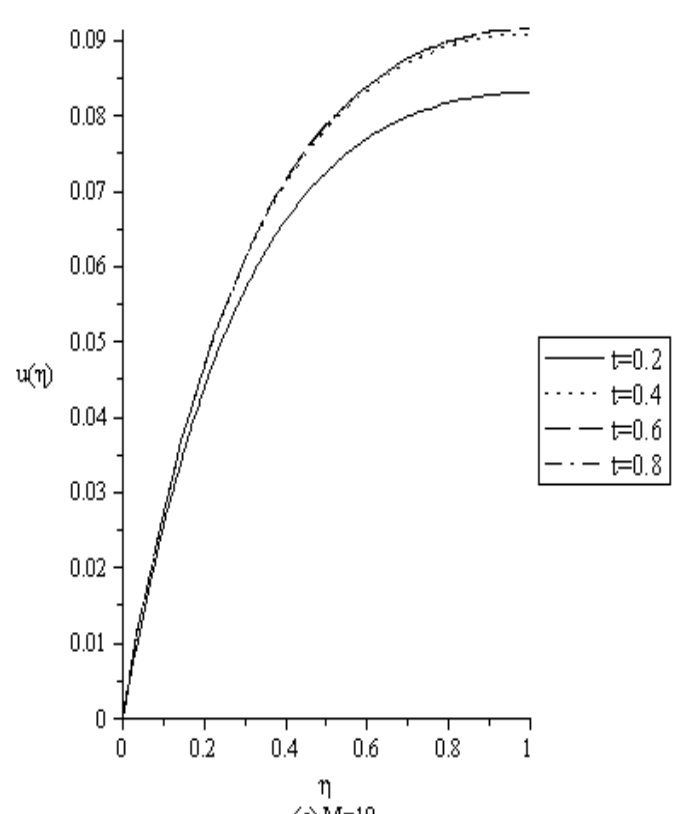

(c) $\mathrm{M}=10$
Figure 1 is evaluated for $K=1, \lambda=0.1$ and $\beta=\varepsilon=0.001$. It is found that velocity profile decrease with increases in magnetic parameter $M$ at large time but increases them at small time. Also it is noticed that at a fixed value of $M, u$ increases with time for short period and then decreases as time develops. However, at large value of $M, u$ attain its
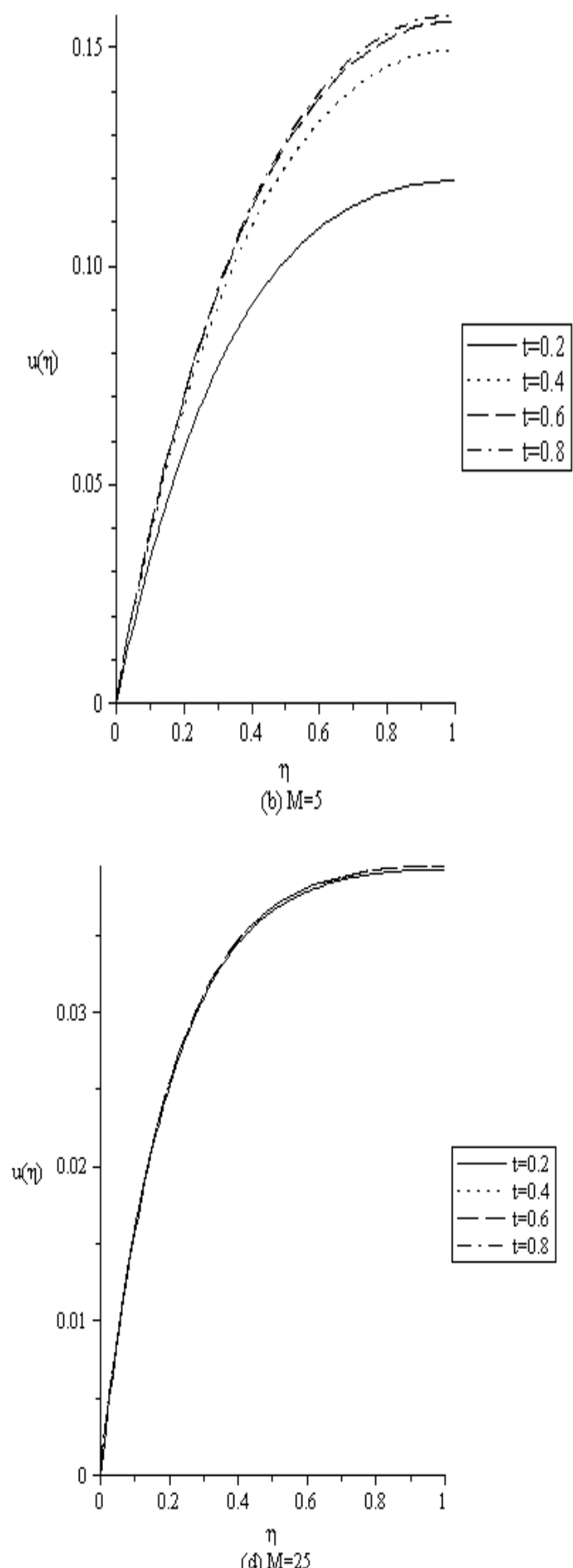

(d) $M=25$

Figure 1: Time development of velocity profile for various values of $M$ and time $t$ when $K=1$ and $\lambda=0.1$. 


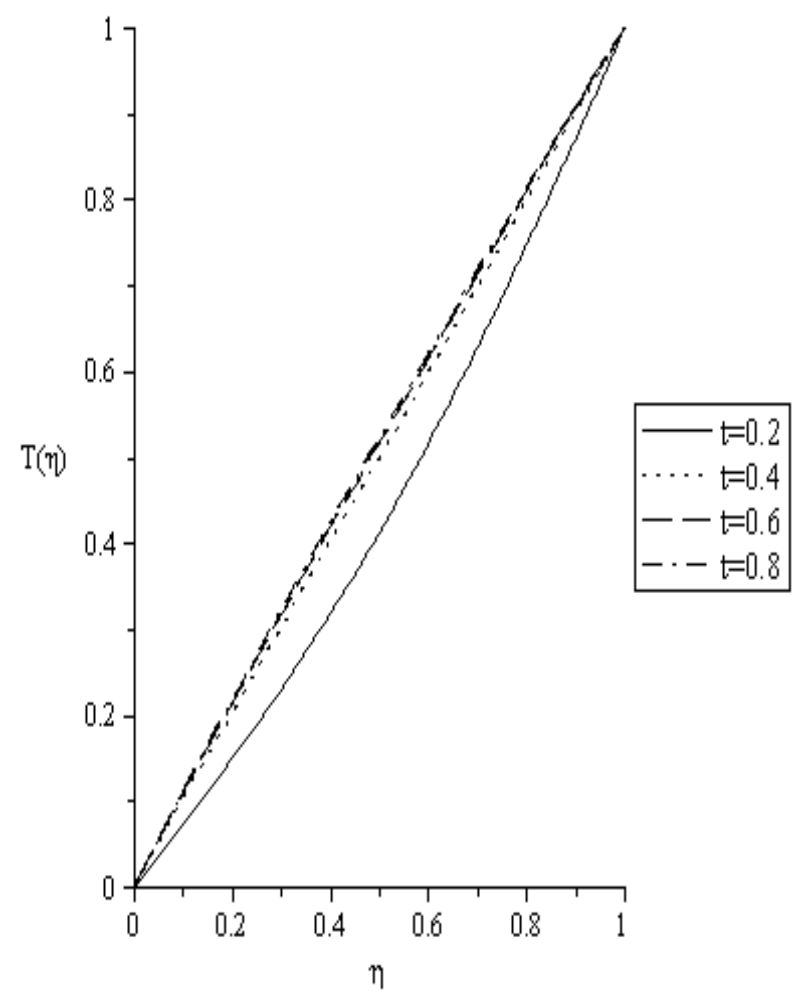

(a) $M=1$

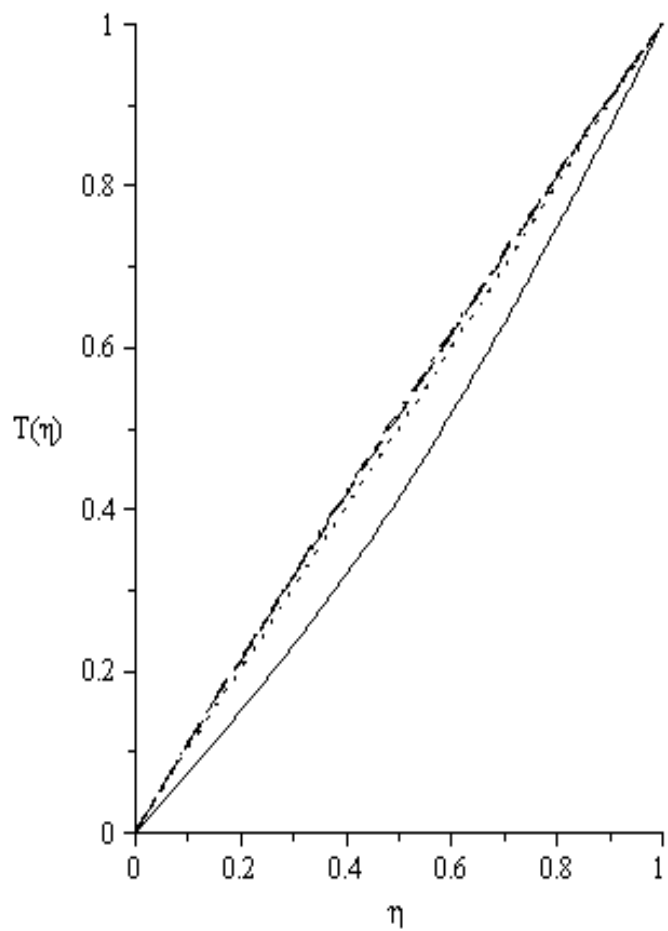

(c) $\mathrm{M}=10$

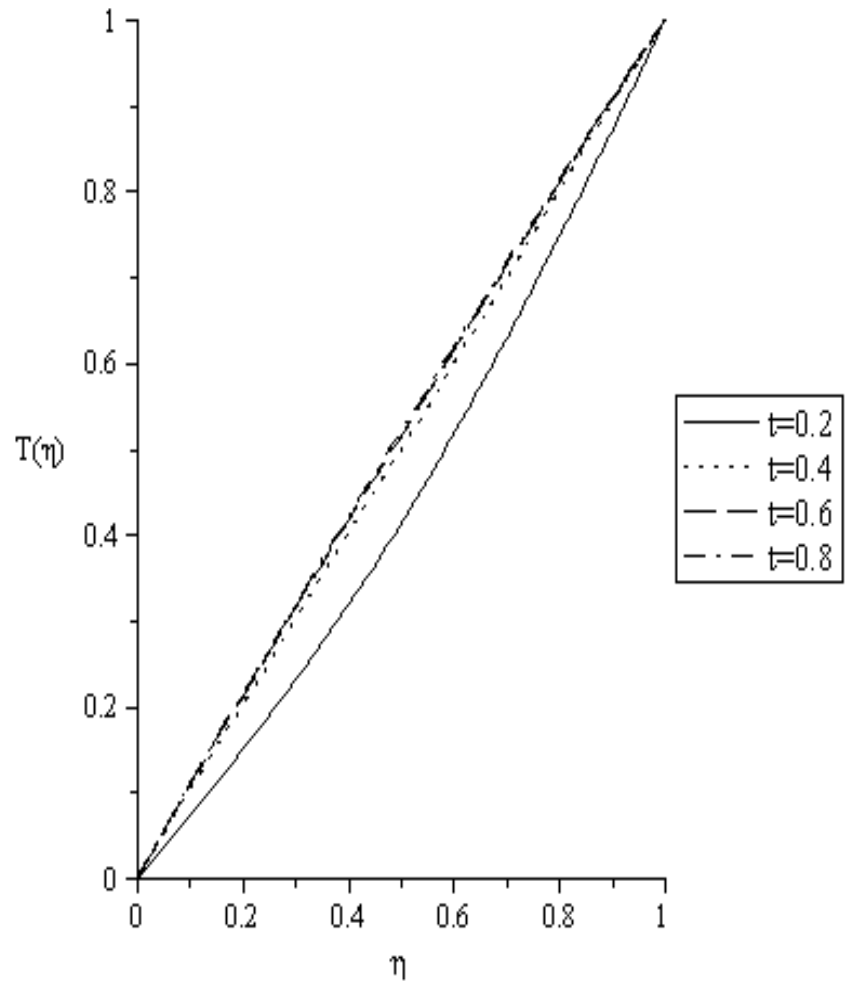

(b) $\mathrm{M}=5$

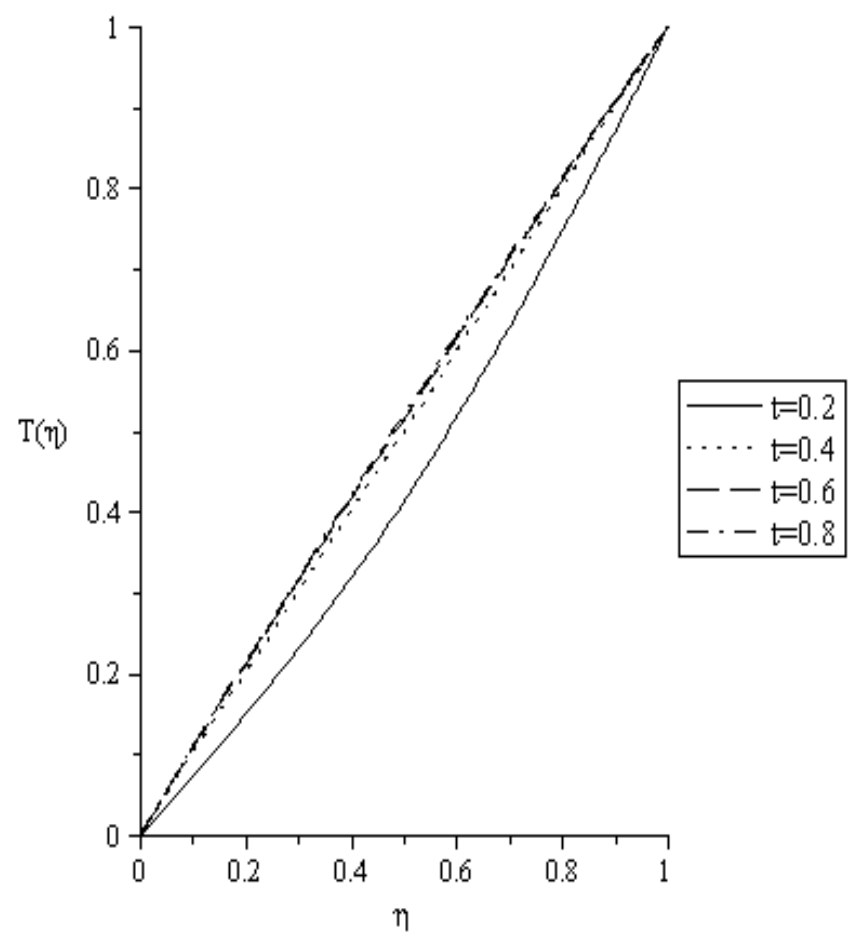

(d) $\mathrm{M}=25$

Figure 2: Time development of temperature profile $T$ for various values of $M$ and time $t$ when $K=1, \lambda=0.1, \operatorname{Pr}=1$ and $E c=1$. 


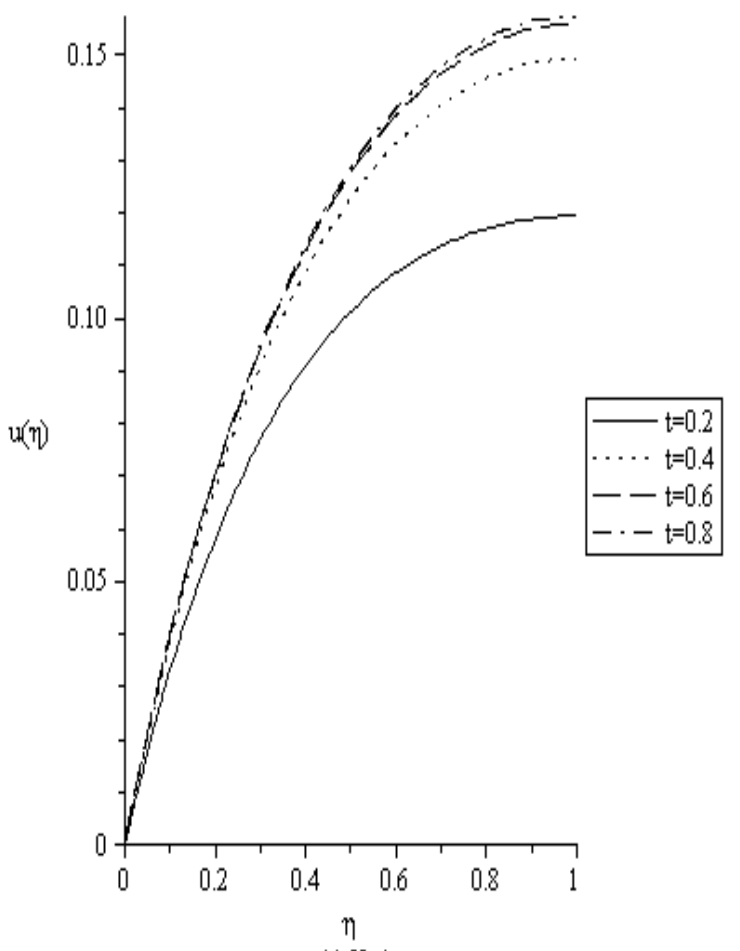

(a) $\mathrm{K}=1$

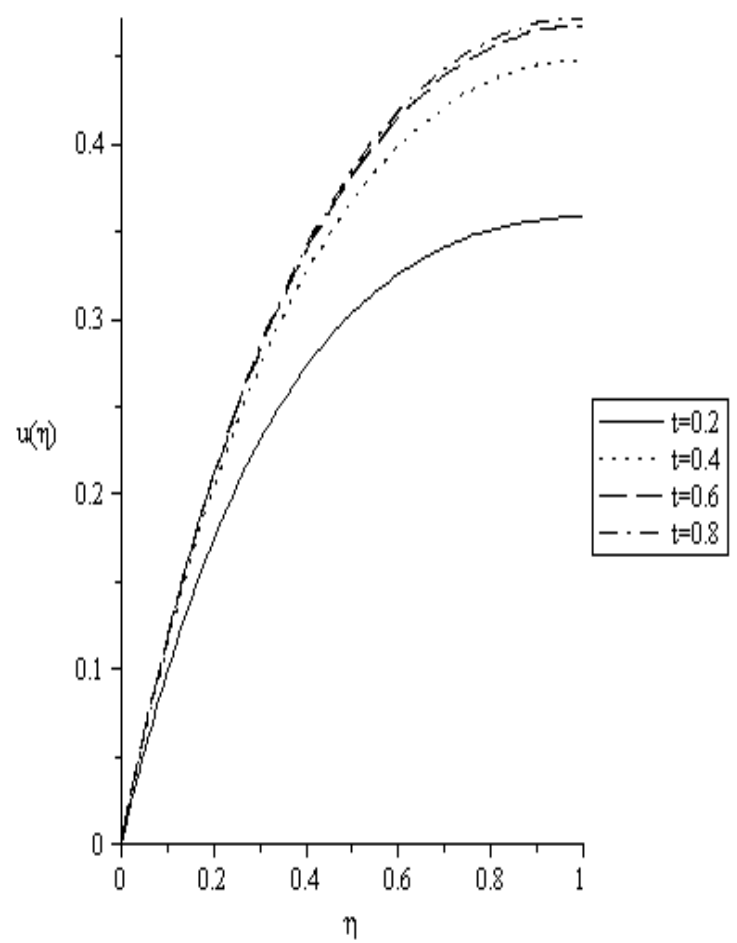

(c) $\mathrm{K}=3$

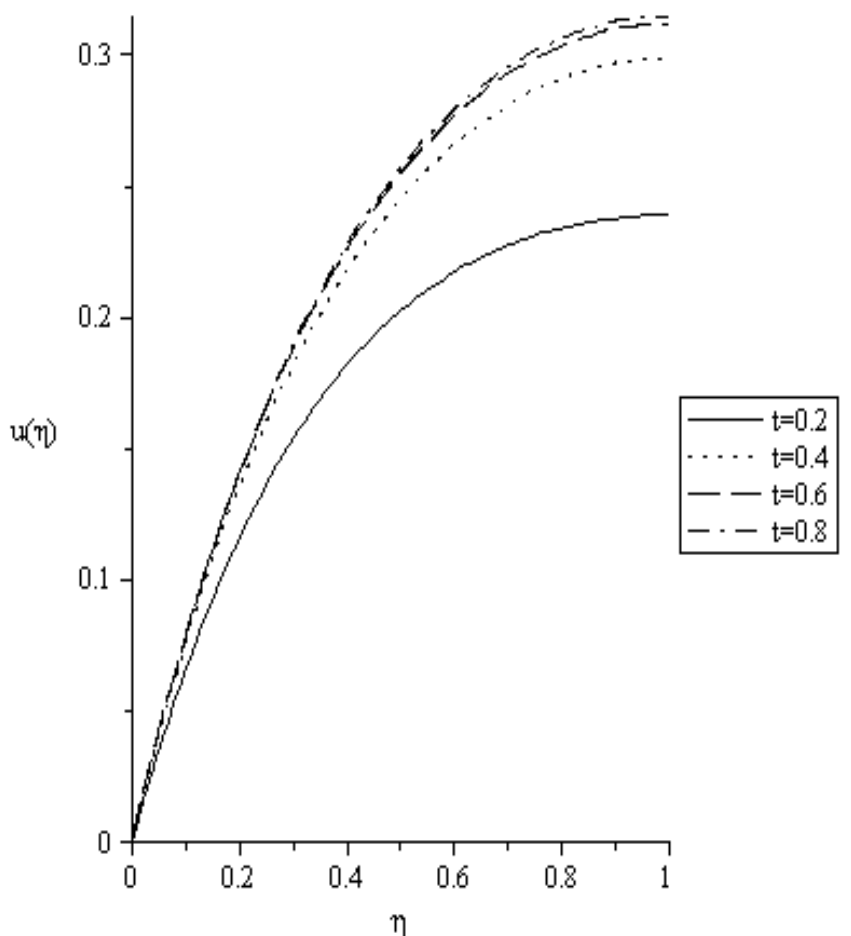

(b) $\mathrm{K}=2$

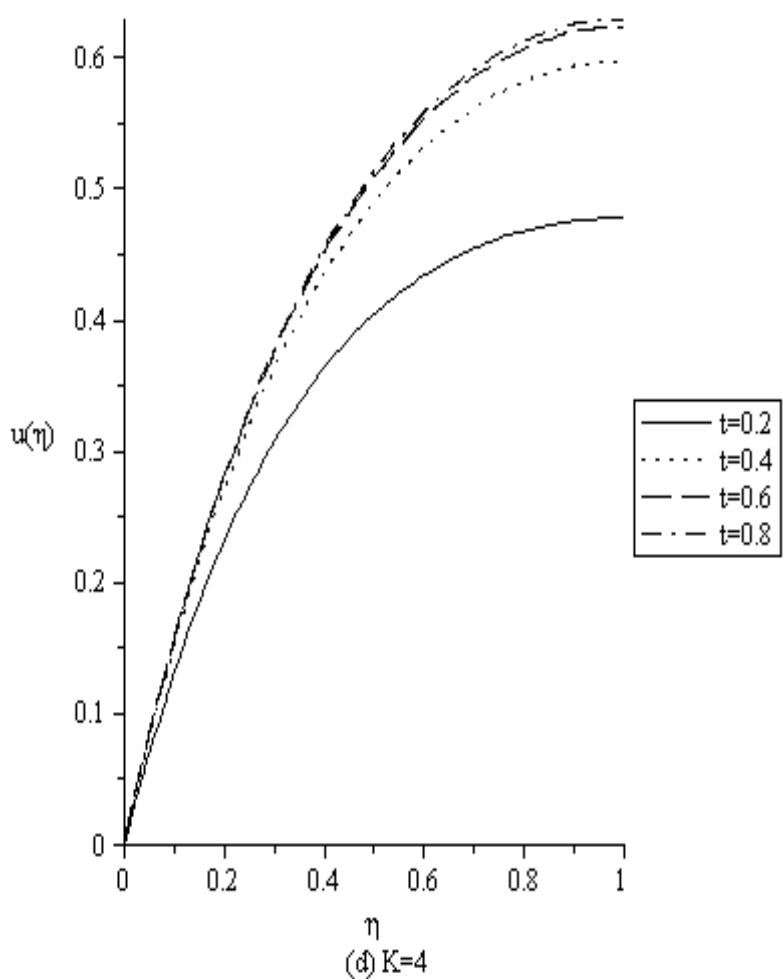

Figure 3: Time development of velocity profile $u$ for various values of $K$ and time $t$ when $M=5$ and $\lambda=0.1$. 


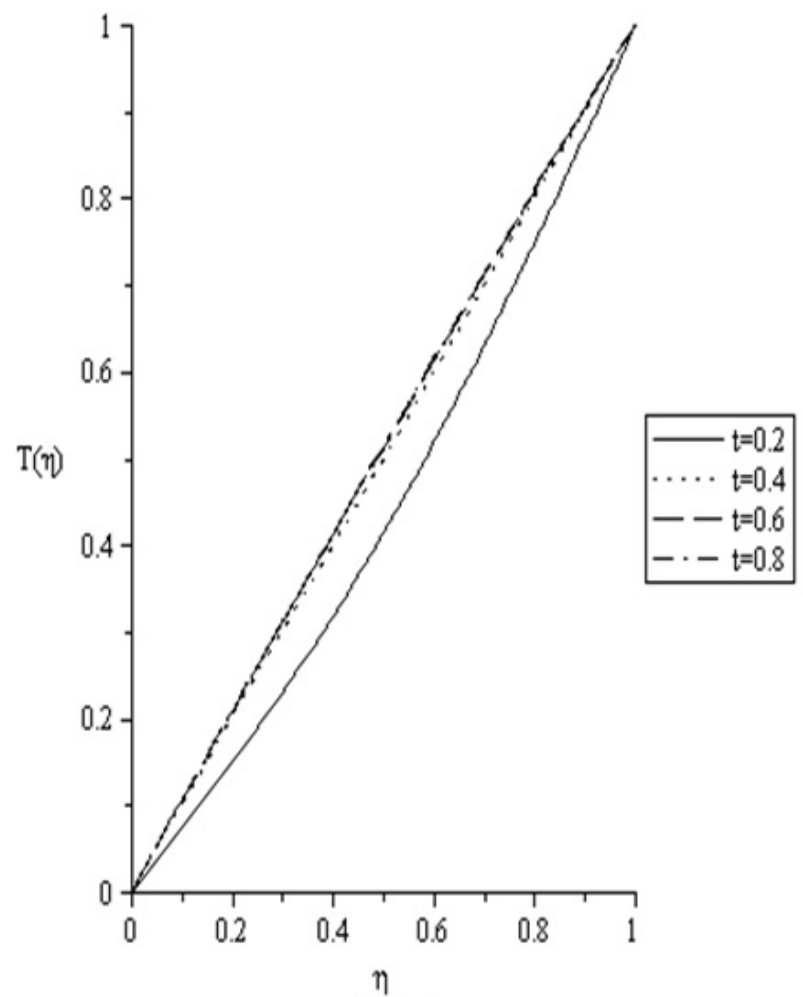

(a) $\mathrm{K}=1$

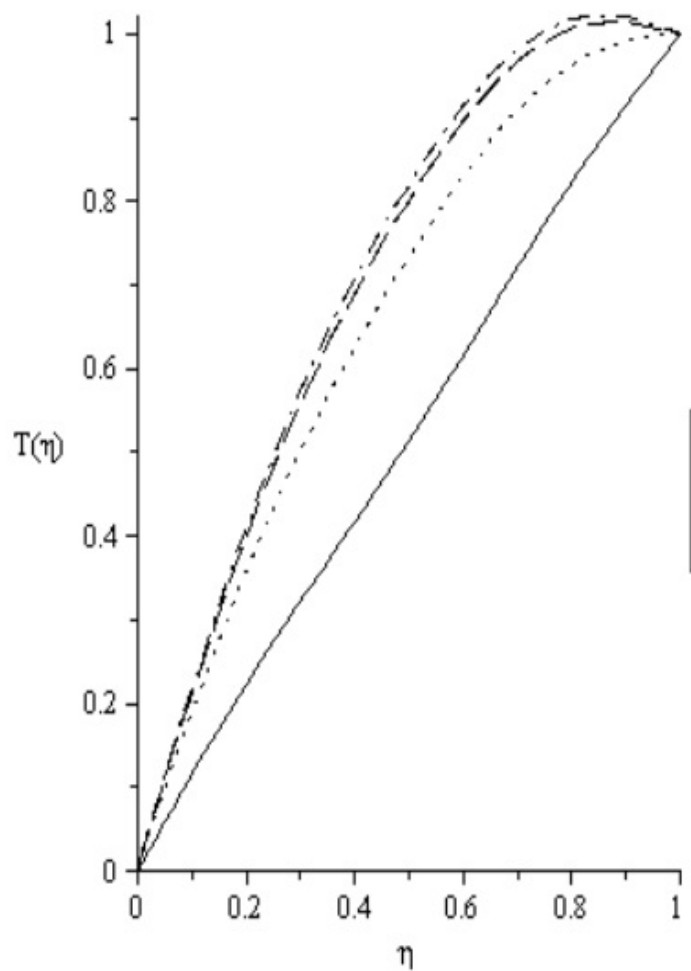

(c) $\mathrm{K}=5$

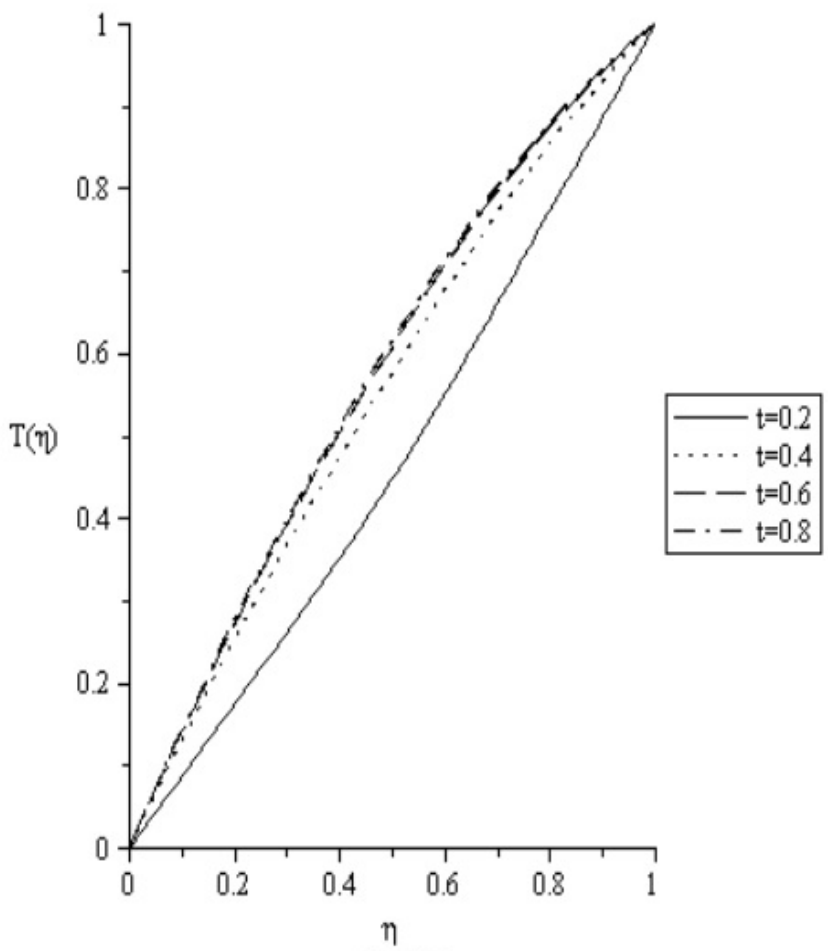

(b) $\mathrm{K}=3$

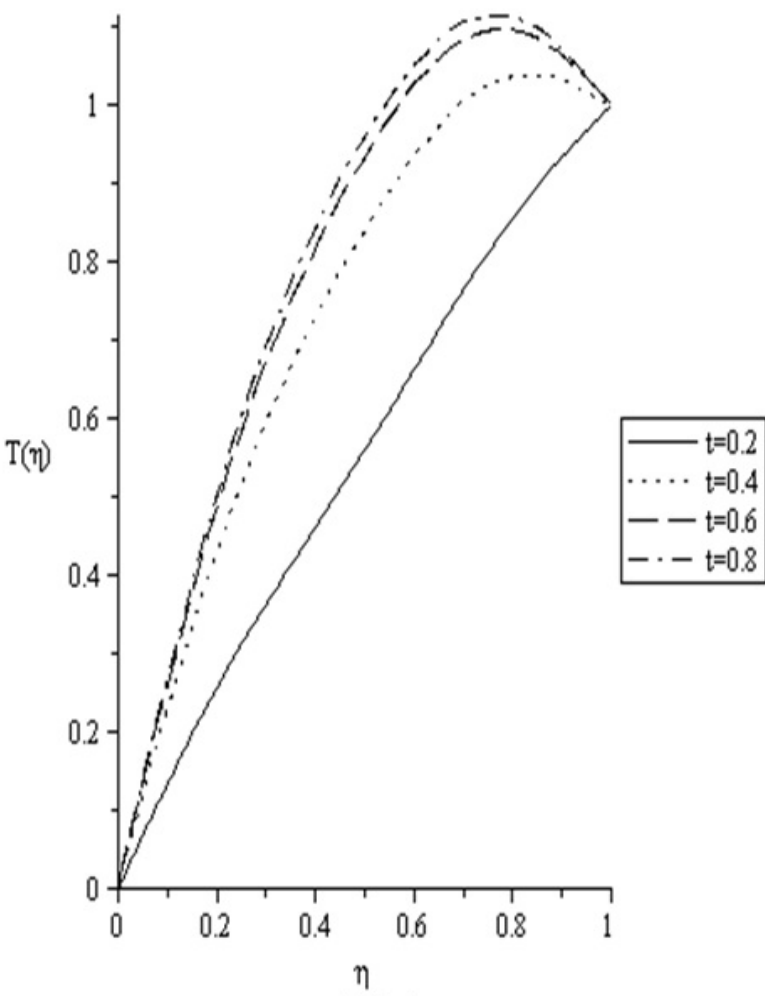

(d) $\mathrm{K}=6$

Figure 4: Time development of temperature profile $T$ for various values of $K$ and time $t$ when $M=5, \lambda=0.1, \operatorname{Pr}=1$ and $E c=1$. 


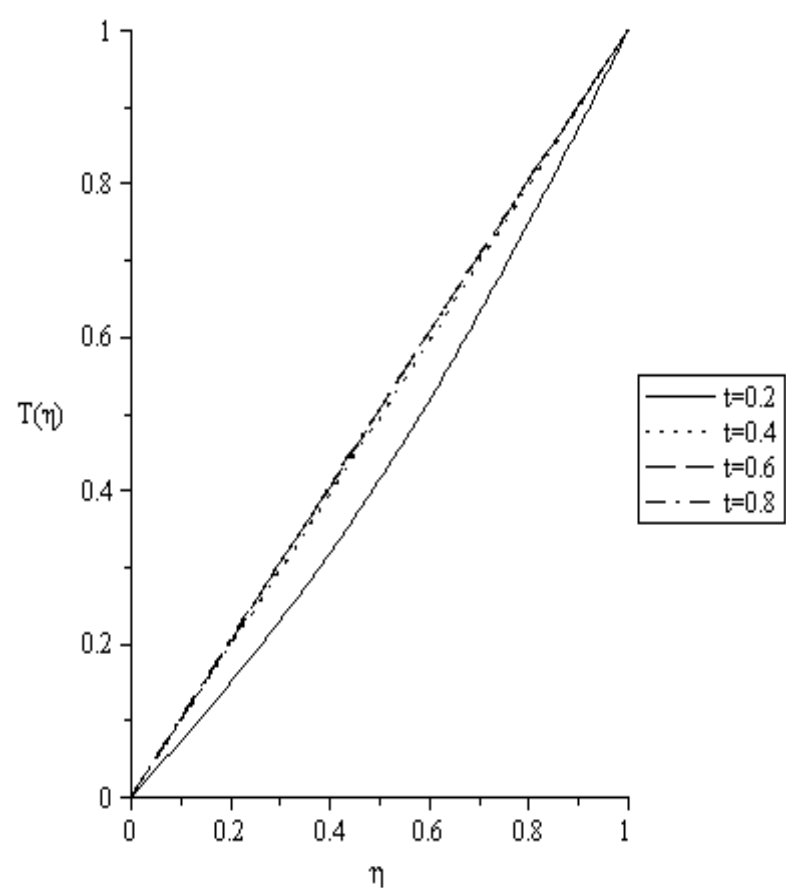

(a) $\mathrm{K}=1$

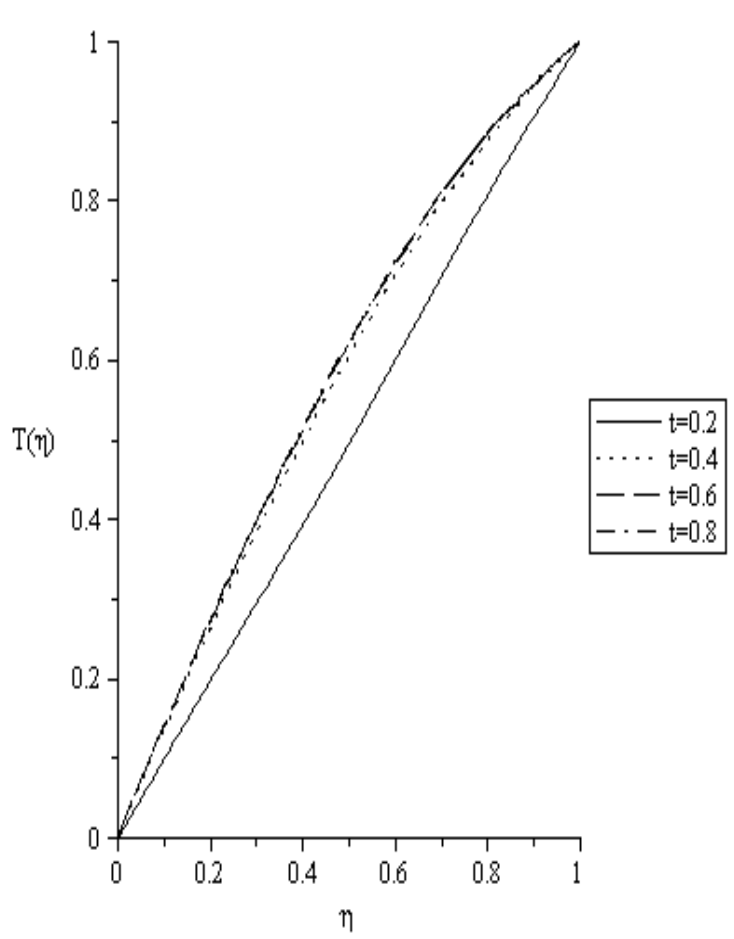

(c) $\mathrm{K}=5$

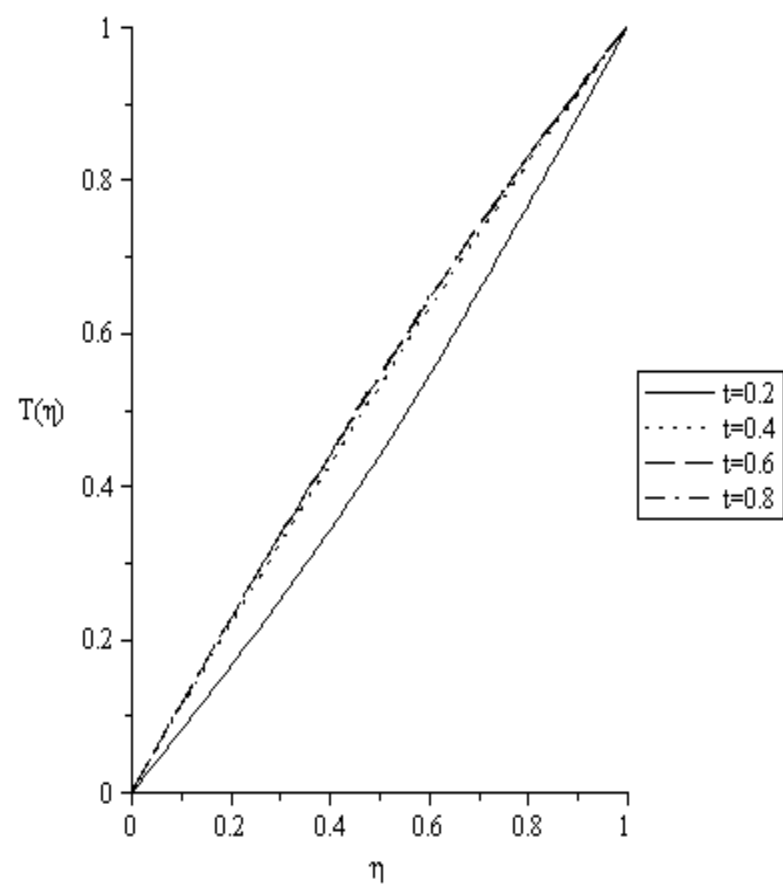

(b) $\mathrm{K}=3$

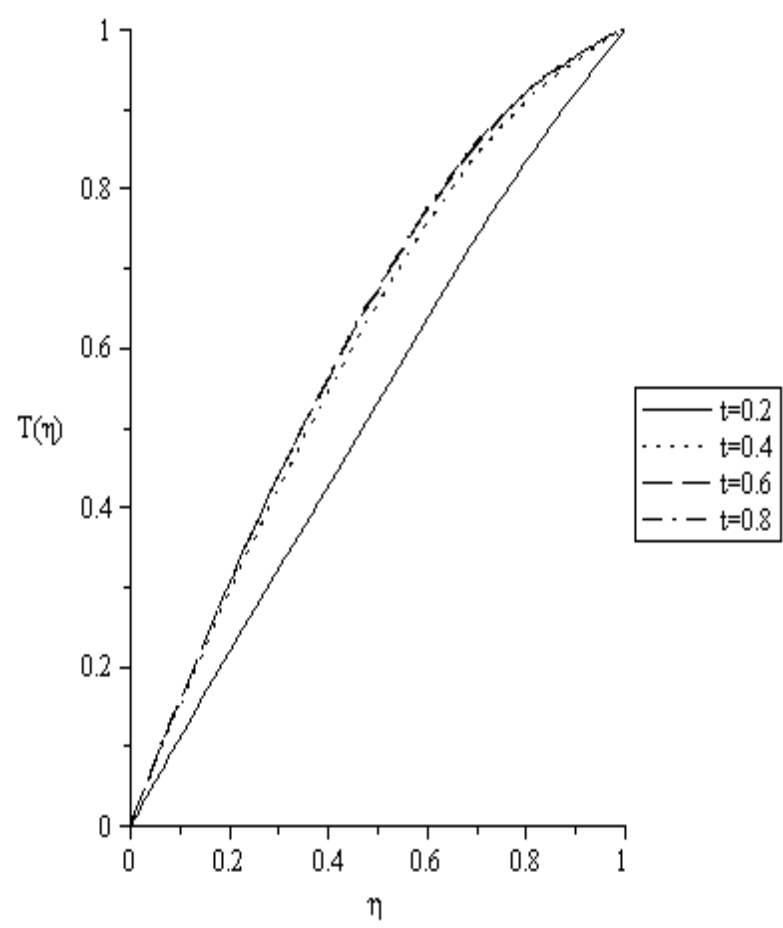

(d) $\mathrm{K}=6$

Figure 5: Time development of temperature profile $T$ for various values of $K$ and time $t$ when $M=20, \lambda=0.1, \operatorname{Pr}=1$ and $E c=1$. 
Citation: Aiyesimi YM, Okedayo GT, Lawal OW (2014) Analysis of Unsteady MHD Thin Film Flow of a Third Grade Fluid with Heat Transfer down an Inclined Plane. J Appl Computat Math 3: 152. doi:10.4172/2168-9679.1000152

Page 11 of 11

steady state monotonically with time as shown in Figure 1d.

Figure 2 is evaluated for $K=1, \lambda=0.1, \beta=0, \operatorname{Pr}=1$ and $E c=1$. It is observed that $T$ slightly increases with time for short period and then remains steady as time developed for various values of $M$. This clearly indicates that the purpose of introducing magnetic field is to decrease the viscous and Joule dissipation as a result of decrease in the velocity profile $u$ and its gradient.

Figures 3 and 4 shows the time development of the velocity and temperature $u$ and $T$ respectively for various values of time $t$ and for gravitational parameter $K=1,2,3$ and 4 . There figure are plotted using $M=5, \lambda=0.1, \operatorname{Pr}=1$ and $E c=1$. It is observed from Figure 3a-3d that for all value of $K, u$ increases at small time but decreases at large time. Also it is shown that the rate at which increases for a short period and the rate at which it decreases as the time develop is the same for each particular value of $K$. This clearly revealed that the effect of gravitational parameter on velocity $K$ depend only on gravitational force and angle of inclination but not on time .

Figure $4 \mathrm{a}-4 \mathrm{~d}$ indicate that the effect of the parameter $K$ on temperature profile $T$ depend not only gravitational force, angle of inclination and magnetic field but also on time $t$. At a small values of $K$ it is observed that $T$ increases slightly at small time but remain steady as time develops. However, when $K$ increase as a result of increase in angle of inclination, $T$ increase substantially at small time and slightly increase at large time. And the rate of increment in $T$ is higher in short period than as time develops for small value of $M$.

This can be attributed to the fact that, for small times, an increase in $K$ increases $u$ largely thereby increase Joule and viscous dissipation at small value of $M$. But at large value of $M$ and increase in $K$ decrease $u$ largely as time develops thereby decreases Joule and viscous dissipation and make $T$ to remain steady as illustrated in Figure 5a-5d.

\section{Conclusion}

The unsteady MHD thin film flow of a third grade fluid down an inclined plane with heat transfer has been studied in the presence of a uniform magnetic field. The effect of magnetic field parameter and gravitational parameter on the velocity and temperature profile has been investigated. It is confirmed that for any value of magnetic field parameter and gravitational parameter, it has a mark effect on steady and unsteady state time of both the velocity and temperature profile. Also, it is interesting to find that the variation of the velocity and temperature profile with the magnetic field parameter and gravitational parameter depends on time.

\section{References}

1. Makinde OD (2009) Thermal critically for a reactive gravity driven thin film flow of a third grade fluid with adiabatic free surface down an inclined plane. App Math Mech Ed 30: 373-380.

2. Hayat T, Saif $S$, Abbas $Z$ (2008) The influence of heart transfer in an MHD second grade fluid film over an unsteady stretching sheet. Physics Letters $A$ 372: 5037-5045.

3. Nayak I, Nayak AK, Padhy S (2012) Numerical solution for the flow and heat transfer of a third grade fluid past a porous vertical plate. Adv Studies Theory Phys 6: 615-624.

4. He JH (1999) Homotopy Perturbation Technique. Comput Math Appl Mech Eng 178: $257-262$.

5. He JH (2003) Homotopy Perturbation Method, a new nonlinear analytical technique. Appl Math Comput 135: 73-79.

6. He JH (2006) Homotopy Perturbation Method for solving boundary value problem. Phys Letter A 350: 87-88.

7. Siddiqui AM, Mahmood R, Ghorn QK (2008) Homotopy Perturbation Method for thin film flow of a third grade fluid down an inclined plane. Chaos solitons and fractals $35: 140-147$.

8. Khan N, Mahmood T (2012) The influence of slip condition on the thin film flow of a third order fluid. International Journal of Non-linear Science 13: 105-116.

9. Aiyesimi YM, Okedayo GT, Lawal OW (2013) "Viscous dissipation effect on the MHD flow of a third grade fluid down an inclined plane with Ohmic heating". Mathematical Theory and Modelling 9: 133-166.

10. Aiyesimi YM, Okedayo GT, Lawal OW (2013) "Unsteady MHD Thin film flow of a third grade fluid with heat transfer and no slip boundary Down an inclined plane". International Journal of Scientific and Engineering Research 4: 420-428.

11. Rivlin RS, Ericksen JL (1955) Stress deformation relations for isotropic materials. J Rat Mech impass Anal 3: 323.

12. Erdogan ME (1995) Plane surface suddenly set into motion in a non-Newtonian fluid. Acta mech 108: 179-187.

13. Asghar S, Gulzar MM, Ayub M (2006) Effects of partial slip on flow of a third grade fluid. Acta Mech Sinica 22: 393-396.

14. Ellehi R (2009) Effects of the slip boundary condition on non-Newtonian flows in a channel. Commu Nonlinear Science Numerical Simul 14: 1377-1384.

15. Sajid M, Awals M, Nadeem S, Hagat T (2008) The influence of slip condition on thin film flow of a fourth grade fluid by the homotopy analysis method. Comput Math Appl 56: 2019-2026. 